\title{
Mucosa-associated bacterial flora of the human colon
}

\author{
I. R. POXTON, R. BROWN, A. SAWYERR* and ANNE FERGUSON*
}

Departments of Medical Microbiology and *Medicine, University of Edinburgh Medical School, Teviot Place, Edinburgh EH8 $9 A G$

\begin{abstract}
Biopsy samples of mucosa were taken during colonoscopy from the proximal colon and rectum of 12 patients, six with ulcerative colitis (UC) and six with non-inflammatory conditions. After anaerobic transport to the laboratory, biopsy specimens were examined by quantitative bacteriological culture on selective and non-selective media for total aerobic count, total anaerobic count, Bacteroides spp., lactobacilli, bifidobacteria and asaccharolytic, lactic acid producers. Isolates of the genus Bacteroides were identified to species level. Counts from proximal colonic and rectal biopsy samples in the same patient were not significantly different. Viable aerobic counts (aerobes and facultative organisms) ranged from $2.4 \times 10^{3}$ to $1.3 \times 10^{6} \mathrm{cfu} / \mathrm{sample}$ biopsy $(5.6 \mathrm{mg})$ and total anaerobic counts were $10-10^{2}$ times higher at $\left(1.4 \times 10^{5}\right)-\left(3 \times 10^{7}\right) \mathrm{cfu} / \mathrm{sample}$. Bacteroides spp. predominated at both sites (range $8.6 \times 10^{4}$ to $1.4 \times 10^{7} \mathrm{cfu} / \mathrm{sample}$ ), comprising $66 \%$ of total counts from proximal colon (range in individual patients 31$80 \%$ ) and $68.5 \%$ from rectum (range 38-91\%). Lactobacilli were isolated from eight biopsy samples from five patients, counts ranging from $3.6 \times 10^{2}$ to $1 \times 10^{5}$ cfu/sample; bifidobacteria were isolated from both sites from 10 of the 12 patients, counts ranging from 50 to $1.8 \times 10^{6} \mathrm{cfu} / \mathrm{sample}$. From the 24 biopsy samples, 235 isolates representing 11 species of Bacteroides were identified. For any individual patient, only a few species (2-7; mean 4.4) of Bacteroides were found, with just one or two species predominating. B. vulgatus was cultured from both samples of seven patients (where it was the major isolate in four) and from single samples of two others; $B$. fragilis was cultured from both sites in six patients, being the major isolate in one patient and second commonest in three, but was not detected in the other six; the majority of other isolates were $B$. merdae/distasonis, B. ovatus, B. thetaiotaomicron and B. uniformis. B. thetaiotaomicron was isolated from both biopsy samples in all three $U C$ patients with active inflammation (16 of the 60 isolates from these patients) but from only four of the other 18 samples from non-inflamed colonic mucosa (nine of 175 isolates).
\end{abstract}

\section{Introduction}

In faeces, strictly anaerobic bacteria outnumber facultative species by a factor of $10^{2}-10^{3}$, with concentrations of anaerobes reaching levels of $10^{10}-10^{12} \mathrm{cfu} / \mathrm{g}$ [1]. The predominant cultivable species in faeces belong to the genus Bacteroides, and the majority of these species are well characterised, belonging to the former ' $B$. fragilis group'. Since the recent changes in classification of the gram-negative anaerobic rods, the intestinal bacteroides represent the entire genus $\mathrm{BaC}$ teroides sensu stricto. B. vulgatus is usually considered

Received 11 June 1996; accepted 14 July 1996.

Corresponding author: Dr I. R. Poxton. one of the most common faecal bacteroides, with $B$. fragilis being one of the less common [2].

Bacteria of the commensal intestinal flora possess a range of important nutritional, immunological, metabolic and pathophysiological properties, and it seems likely that those in the immediate vicinity of the mucosal surface will be particularly relevant to some of these functions. In contrast to an enormous amount of literature on the bacteria of faeces, there are few comprehensive published data on the quantitative bacteriology of the mucosa-associated bacteria of the human colon. Most quantitative studies, e.g., those of Peach and Tabaqchali [3], Hudson et al. [4], Hudson [5] and Hartley et al. [6] did not identify bacteria to species level. For colonic mucosa-associated counts, the reported difference between strict anaerobes and 
facultative organisms is not of the same order as in faeces, the ratio being nearer unity, with some studies showing anaerobes outnumbering facultative organisms by a factor of between 5 (biopsy samples) and 40 (resected samples) [5]. There is a single report by Namavar et al. [2], in which the colonic luminal and mucosa-adherent Bacteroides species in 10 patients with colon cancer were assessed quantitatively in faeces, gut lavage fluid and biopsy samples from operative resection specimens. B. fragilis was found with a low frequency in the faeces (only $4 \%$ of the total Bacteroides spp.), but had a more intimate association with the gut mucosa than other Bacteroides spp. representing $42 \%$ of the total bacteroides. The authors suggested that this might explain why $B$. fragilis is commonly isolated from patients with gutassociated abdominal sepsis.

In the present study, endoscopic biopsy samples from the proximal colon and rectum of 12 patients (six with ulcerative colitis) were examined by quantitative aerobic and anaerobic culture and the individual Bacteroides species in each patient were identified.

\section{Materials and methods}

\section{Patients and specimens}

Endoscopic biopsy samples from 12 patients (seven men and five women; age range 21-67 years, mean 48) in whom colonoscopy was being done in the course of routine clinical care were examined. Six had chronic ulcerative colitis (UC); this was clinically, endoscopically and histologically inactive in three cases, and three patients had active disease with mucosal inflammation. Two UC patients, both with inactive disease, were taking maintenance sulphasalazine (containing sulphapyridine). Of the remaining six patients, three had previous colonic neoplasms, two had irritable bowel syndrome and one had diverticulosis. Because, with one exception discussed below, there were no differences in the bacteriological findings in UC and other patients, data for all 12 patients are presented together.

Preparation for colonoscopy was as follows: clear fluids only by mouth for $48 \mathrm{~h}$ before the procedure; co-danthrusate $50 / 60$, two capsules $60 \mathrm{~h}$ before and two capsules $36 \mathrm{~h}$ before the procedure; and Picolax (Ferring Pharmaceuticals, Feltham, Middlesex) one sachet $24 \mathrm{~h}$ and one sachet $16 \mathrm{~h}$ before the procedure. Colonoscopy was performed with midazolam and pethidine sedation; instruments and biopsy forceps were cleansed with glutaraldehyde (Cidex: Johnson and Johnson, Skipton, Yorkshire). Forceps were rinsed in sterile saline before sampling. In each patient, one biopsy sample was taken from the proximal colon (ascending colon in 10, transverse colon in two) and one from the rectum. The mucosal samples were immediately (within $30 \mathrm{~s}$ ) transferred with a sterile needle from the forceps into the transport system (see below). Previously, a separate evaluation had shown that the weight of 21 biopsy samples taken with the standard forceps was reasonably consistent $(5.6 \mathrm{mg}$, SD $1.6 \mathrm{mg}$ ).

This use of colonic mucosal biopsies was approved by the Lothians Medicine and Medical Oncology Ethics Committee.

\section{Processing of biopsy material and quantitative bacteriology}

Biopsy samples were transported to the laboratory within 45-60 min after collection in the Anatube type II anaerobic transport system [7] containing $0.2 \mathrm{ml}$ of Stuart's transport medium (Unipath) supplemented with yeast extract (Unipath) $0.5 \%$. In the laboratory, the whole transport system was placed inside an anaerobic cabinet $\left(\mathrm{N}_{2} 80 \%, \mathrm{H}_{2} \quad 10 \%, \mathrm{CO}_{2} 10 \%\right)$ and the biopsy sample and the $0.2 \mathrm{ml}$ of transport medium were transferred into a sterile 'Stomacher' bag, together with $1.8 \mathrm{ml}$ of pre-reduced anaerobe investigation medium (AIM) [8]. The sample was broken up by squeezing it manually through the bag, and then the suspension was vigorously aspirated into and out of a Pasteur pipette to ensure complete homogenisation. For anaerobic incubation, 10-fold dilutions were made in pre-reduced AIM, 20- $\mu$ l volumes were seeded on to the culture plates and the inoculum was spread with a sterile glass spreader. Portions of the relevant dilutions were transferred out of the cabinet to inoculate plates for aerobic incubation. After incubation at $37^{\circ} \mathrm{C}$, plates were examined and colonies were counted after 2-3 days and again after 7 days. The total anaerobic counts (as indicated in Table 1) included strict anaerobes and facultative organisms.

The following media were used: Columbia Agar (Unipath) supplemented with horse blood 5\% for total counts of aerobic and facultative organisms (BA); Fastidious Anaerobe Agar (Lab M) supplemented with horse blood $4 \%$ and freeze-thaw lysed horse blood $1 \%$ for total anaerobic counts (FAA); FAA supplemented with kanamycin $75 \mathrm{mg} / \mathrm{L}$ and vancomycin $2.5 \mathrm{mg} / \mathrm{L}$ for Bacteroides spp. (FAAKV); Rogosa Agar (Unipath) for Lactobacillus spp. (RA); Reinforced Clostridial Cotton Blue Agar [9] for Bifidobacterium spp. (RCCBA).

\section{Identification of isolates}

This was done initially by examination of colonial morphology on primary isolation plates and cell morphology in gram-stained films. Isolates from RA and RCCBA that were gram-positive rods were subcultured to AIM broth containing glucose $1 \%$ and the resultant culture was analysed by gas chromatography for short-chain fatty acids [8]. Isolates that produced only lactic acid in major amounts were 
Table 1. Summary of quantitative bacteriology of 24 biopsy samples from 12 patients

\begin{tabular}{|c|c|c|c|c|c|c|c|}
\hline \multirow[b]{2}{*}{ Diagnosis } & \multirow[b]{2}{*}{$\begin{array}{l}\text { Biopsy } \\
\text { site }\end{array}$} & \multicolumn{6}{|c|}{ Viable counts (cfu/sample) } \\
\hline & & $\begin{array}{c}\text { Total aerobic } \\
\text { count }\end{array}$ & $\begin{array}{c}\text { Total anaerobic } \\
\text { count }\end{array}$ & $\begin{array}{l}\text { Bacteroides } \\
\text { spp. }\end{array}$ & Lactobacilli & Bifidobacteria & $\begin{array}{c}\text { Asaccharolytic } \\
\text { lactic acid } \\
\text { producers }\end{array}$ \\
\hline \multirow[t]{2}{*}{ UC active } & $\mathrm{AC}$ & $1.4 \times 10^{5}$ & $9.4 \times 10^{5}$ & $7.4 \times 10^{5}$ & - & $1.4 \times 10^{3}$ & - \\
\hline & $\mathrm{R}$ & $2.4 \times 10^{5}$ & c. $9.0 \times 10^{6}$ & $c .8 .6 \times 10^{6}$ & c. $1.0 \times 10^{5}$ & c. $6.4 \times 10^{4}$ & _- \\
\hline \multirow[t]{2}{*}{ UC active } & $\mathrm{AC}$ & $3.8 \times 10^{4}$ & $2.4 \times 10^{6}$ & $1.5 \times 10^{6}$ & $1.1 \times 10^{3}$ & c. $4.2 \times 10^{4}$ & - \\
\hline & $\mathrm{R}$ & $2.4 \times 10^{5}$ & $9.2 \times 10^{6}$ & $5.8 \times 10^{6}$ & $2.2 \times 10^{3}$ & c. $1.2 \times 10^{5}$ & - \\
\hline \multirow{2}{*}{ UC active } & PTC & $4.8 \times 10^{4}$ & $8.6 \times 10^{5}$ & $7.0 \times 10^{5}$ & - & $1.0 \times 10^{2}$ & - \\
\hline & $\mathrm{R}$ & $3.0 \times 10^{3}$ & $1.4 \times 10^{5}$ & $8.6 \times 10^{4}$ & - & $5.0 \times 10^{1}$ & - \\
\hline \multirow[t]{2}{*}{ UC inactive } & $\mathrm{AC}$ & $4.0 \times 10^{4}$ & $3.2 \times 10^{6}$ & $2.2 \times 10^{6}$ & - & - & - \\
\hline & $\mathrm{R}$ & $6.0 \times 10^{4}$ & $9.0 \times 10^{6}$ & $6.2 \times 10^{6}$ & - & - & - \\
\hline \multirow[t]{2}{*}{$\mathrm{UC}$ inactive } & $\mathrm{AC}$ & $3.0 \times 10^{4}$ & $7.8 \times 10^{6}$ & $2.4 \times 10^{6}$ & _- & $1.9 \times 10^{6}$ & $\ldots$ \\
\hline & $\mathrm{R}$ & $2.2 \times 10^{4}$ & $3.8 \times 10^{6}$ & $1.4 \times 10^{6}$ & - & $9.8 \times 10^{3}$ & - \\
\hline \multirow[t]{2}{*}{ UC inactive } & $\mathrm{AC}$ & $1.8 \times 10^{5}$ & $2.4 \times 10^{6}$ & $1.6 \times 10^{6}$ & -- & $6.0 \times 10^{2}$ & - \\
\hline & $\mathrm{R}$ & $1.3 \times 10^{5}$ & $1.1 \times 10^{6}$ & $5.4 \times 10^{5}$ & _- & $6.0 \times 10^{2}$ & _- \\
\hline \multirow[t]{2}{*}{ DD } & $\mathrm{AC}$ & c. $1.3 \times 10^{6}$ & $c .4 .0 \times 10^{6}$ & c. $2.0 \times 10^{6}$ & - & $5.0 \times 10^{4}$ & - \\
\hline & $\mathrm{R}$ & $1.4 \times 10^{5}$ & $5.6 \times 10^{5}$ & $2.8 \times 10^{5}$ & - & $9.8 \times 10^{3}$ & - \\
\hline \multirow[t]{2}{*}{ IBS } & $\mathrm{AC}$ & $1.6 \times 10^{5}$ & $1.1 \times 10^{6}$ & $8.6 \times 10^{5}$ & _- & $1.5 \times 10^{5}$ & - \\
\hline & $\mathrm{R}$ & $4.8 \times 10^{4}$ & $2.2 \times 10^{5}$ & $1.6 \times 10^{5}$ & - & $4.0 \times 10^{4}$ & - \\
\hline \multirow[t]{2}{*}{ IBS } & $\mathrm{AC}$ & $8.8 \times 10^{3}$ & $1.5 \times 10^{6}$ & $1.2 \times 10^{6}$ & - & $4.6 \times 10^{4}$ & - \\
\hline & $\mathrm{R}$ & $5.4 \times 10^{3}$ & $1.5 \times 10^{6}$ & $8.4 \times 10^{5}$ & $3.8 \times 10^{4 *}$ & $9.0 \times 10^{3}$ & _- \\
\hline \multirow[t]{2}{*}{ Previous $\mathrm{Ca} \mathrm{AC}$} & PTC & c. $7.6 \times 10^{5}$ & $5.2 \times 10^{6}$ & $3.4 \times 10^{6}$ & $1.0 \times 10^{4}$ & $2.8 \times 10^{3}$ & $2.4 \times 10^{3}$ \\
\hline & $\mathrm{R}$ & $6.2 \times 10^{5}$ & $6.0 \times 10^{6}$ & $2.6 \times 10^{6}$ & $2.2 \times 10^{4}$ & $5.6 \times 10^{3}$ & c. $6.0 \times 10^{4}$ \\
\hline \multirow[t]{2}{*}{ Previous $\mathrm{Ca} \mathrm{AC}$} & $\mathrm{AC}$ & $3.6 \times 10^{5}$ & $3.0 \times 10^{7}$ & $1.4 \times 10^{7}$ & $1.1 \times 10^{3}$ & $1.9 \times 10^{3}$ & - \\
\hline & $\mathrm{R}$ & $1.1 \times 10^{5}$ & $9.4 \times 10^{6}$ & $8.2 \times 10^{6}$ & $3.6 \times 10^{2}$ & $3.0 \times 10^{2}$ & - \\
\hline \multirow[t]{2}{*}{ Adenoma Fup } & $\mathrm{AC}$ & $2.8 \times 10^{3}$ & $1.4 \times 10^{6}$ & $1.1 \times 10^{6}$ & - & - & $c .7 .0 \times 10^{3}$ \\
\hline & $\mathrm{R}$ & $2.4 \times 10^{3}$ & $1.6 \times 10^{6}$ & $1.3 \times 10^{6}$ & - & _- & c. $3.2 \times 10^{3}$ \\
\hline
\end{tabular}

UC, ulcerative colitis; DD, diverticulitis disease; IBS, irritable bowel syndrome; $\mathrm{Ca} A \mathrm{AC}$, cancer, ascending colon; Fup, follow up; AC, ascending colon; $R$, rectum, PTC, proximal transverse colon; -, not isolated, $<50 \mathrm{cfu} / \mathrm{sample}$.

* May be bifidobacteria.

considered to be Lactobacillus spp. and those that produced major amounts of acetic and lactic acids in a ratio of $>1: 1$ were considered to be Bifidobacterium spp. Colonies that were Bacteroides-like $(0.5-4 \mathrm{~mm}$, round and entire, convex, smooth or mucoid, shiny, translucent or opaque, grey, haemolytic or nonhaemolytic) from FAAKV and were gram-negative bacilli or cocco-bacilli were subcultured to AIMcooked meat broth and identified [8]. Ten such colonies were selected from the dilution that gave 100-200 colonies on the FAAKV plate for identification to species level.

\section{Results}

There were no significant differences between the counts from proximal colonic and rectal biopsy samples from the same patient. Aerobic viable counts ranged from $2.4 \times 10^{3}$ to $1.3 \times 10^{6} \mathrm{cfu} / \mathrm{sample}$, and total anaerobic counts (essentially, total counts) 10 $10^{2}$-fold higher at $1.4 \times 10^{5}$ to $3.0 \times 10^{7} \mathrm{cfu} / \mathrm{sample}$ (Table 1). Facultative anaerobes (essentially Escherichia coli and other coliforms) were included in both counts. In most cases, where the difference in counts was $\geqslant 10$-fold, the total aerobic count was equivalent to the $E$. coli count and the total anaerobic count represents strict anaerobes. However, where the counts are of a similar order of magnitude, a significant proportion of the anaerobic count will be made up of facultative organisms.
Bacteroides spp. predominated in the flora at both sampling sites (range $8.6 \times 10^{4}$ to $1.4 \times 10^{7} \mathrm{cfu} /$ sample), comprising $66 \%$ of the total anaerobic counts from proximal colon (range in individual patients $31-$ $80 \%$ ) and $68.5 \%$ of total anaerobic counts (range $38-$ $91 \%$ ) from rectum. Lactobacilli were isolated from eight biopsy samples from five patients, with counts in the range $\left(3.6 \times 10^{2}\right)-\left(1 \times 10^{5}\right) \mathrm{cfu} / \mathrm{sample}$. Bifidobacteria were isolated from both samples taken from 10 of the 12 patients, with counts in the range 50$\left(1.9 \times 10^{6}\right) \mathrm{cfu} /$ sample. Asaccharolytic lactic acid producers were isolated in low numbers $-\left(2.4 \times 10^{3}\right)$ $\left(6 \times 10^{4}\right) \mathrm{cfu} /$ sample - from only two patients, neither of whom had inflammatory conditions.

From the 24 biopsy samples, 235 isolates representing 11 species of Bacteroides were identified (Table 2). Generally, there was concordance between the proximal colon and rectum, as to the presence or absence of a particular species. For any individual patient, only a few species (2-7: mean 4.4) of Bacteroides were found, with just one or two species predominating. $B$. vulgatus was cultured from both samples of seven patients (where it was the major isolate in four) and from single biopsy samples of two others. B. fragilis was cultured from both sites in six of the 12 patients, being the major isolate in one patient and the second most frequent in three, but was not detected in the other six. The majority of other isolates were $B$. merdae/distasonis, B. ovatus, B. thetaiotaomicron or B. uniformis. 


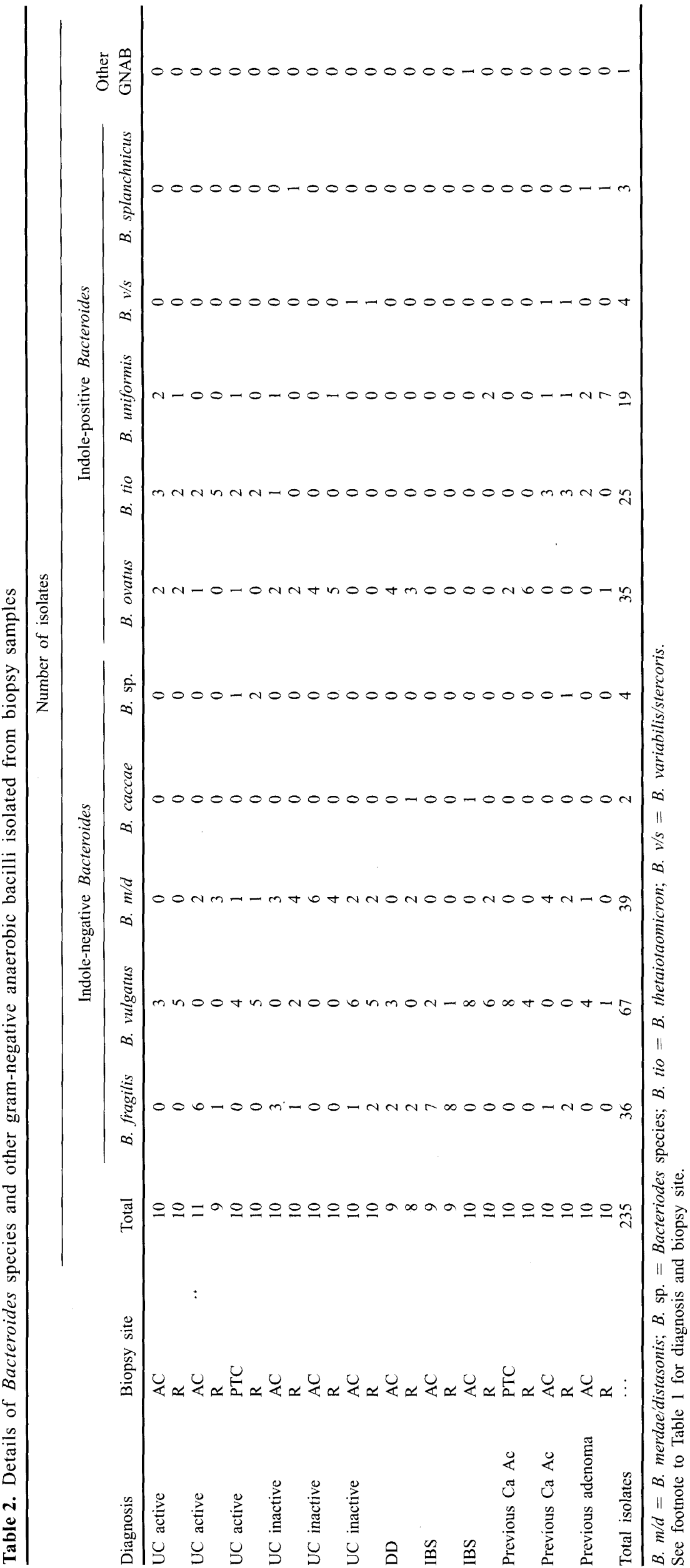


B. thetaiotaomicron was isolated from both biopsy samples from all three UC patients with active inflammation (16 of the 60 isolates from these patients) but from only four of the other 18 biopsy samples from non-inflamed colonic mucosa (nine of the $175^{\circ}$ isolates).

\section{Discussion}

It is likely that mucosal adherence of colonic bacteria is influenced by factors within the lumen, properties of the organisms themselves and the local immune response. Many of the bacteria in the gastrointestinal tract, especially the gram-negative rods, are opportunist pathogens and are capable of eliciting injurious, proinflammatory responses if viable bacteria or soluble cell-surface components interact with cells of the systemic immune system. Therefore, it is important to know which species are in the most intimate association with the mucosa, and how the immune response to these species is mounted and controlled.

There are probably several reasons why there are few published data on this important topic. Faeces, and the contents of the intestinal lumen, are readily available for study whereas there are logistic and ethical difficulties in obtaining fresh biopsy tissue to allow investigation of the mucosal flora. In the past, tissue has become available mainly as operative specimens $[2,5]$. In these, the microbial flora may have been influenced by pre-operative preparation which would normally include starvation, bowel cleansing and often antibiotic treatment pre-operatively; intra-operatively, oxygenation and viability of the colon will have been influenced by the operative technique and, in particular, the amount of time between interruption of the arterial supply and removal of the specimen from the body to allow samples to be collected for study.

The size of the clinical specimen, resected material or biopsy, varies greatly, and in most studies, the number of bacteria is related to weight (of sample) as the unit of measurement, even though surface area is much more appropriate. In previous studies, specimens have varied from $c .3 \mathrm{~cm}^{2}$ of visually normal colon dissected from resected specimens [2], to c. 5-mg biopsy samples [3]. By weight, mean total counts obtained were up to $6 \times 10^{7} \mathrm{cfu} / \mathrm{g}$ of tissue [2], but most studies have reported counts of around $10^{6} \mathrm{cfu} / \mathrm{g}$ $[3,6]$. If the results of the present study were expressed per gram of tissue, the counts would be up to $5 \times 10^{9} \mathrm{cfu} / \mathrm{g}$. Even acknowledging the possible errors of using counts/unit weight, the techniques employed in the present study appear to have achieved much higher isolation rates than those of other studies. Nevertheless the ideal denominator is probably mucosal surface area rather than biopsy weight. The surface area of the biopsy samples in this study was estimated as $20 \mathrm{~mm}^{2}$.

The proportions of different Bacteroides spp. identified in this study were different from those reported in the only other study where Bacteroides spp. were identified, especially in regard to $B$. fragilis. This comparison is summarised in Table 3 , but it should be noted that the study by Namavar et al. was performed on colonic mucosal specimens obtained at operation.

Preparation for colonoscopy has the potential to influence the colonic flora. Patients in this study were given stimulant laxative treatment for 3 days and a fluid diet for 2 days before colonoscopy. Nevertheless the observation that the mucosa-associated flora of the rectum and the proximal colon are similar indicates that valuable information may be obtained from rectal or sigmoid biopsy samples which are readily collected from an unprepared colon. Although two of the UC patients in the present study were receiving sulphasalazine, a recent study on the use of this drug in the treatment of UC concluded that it had little effect on the bacteria colonising the colorectal mucosa [6].

In anaerobic infections outside the gastrointestinal tract it is well established that certain species of Bacteroides are much more likely to be isolated than others, and this has been related to the relative virulence, or pathogenic potential of the species. $B$. fragilis is the Bacteroides species most commonly isolated from clinical specimens, ranging from $78 \%$ in

Table 3. Comparison of results with those of Namavar et al. [2]

\begin{tabular}{lccc}
\hline & \multicolumn{2}{c}{ Percentage of Bacteroides isolates } \\
\cline { 2 - 3 } Bacteroides & Namavar et al. [2] & $\begin{array}{c}\text { This study } \\
\text { (mucosa) }\end{array}$ \\
\cline { 2 - 4 } species & Faeces & Mucosa & 15.6 \\
\hline B. fragilis & 4.4 & 42.8 & 28.1 \\
B. vulgatus & 45 & 25.6 & 16.9 \\
B. distasonis & 9.3 & 9.8 & 7.4 \\
B. uniformis & 22.2 & 10.3 & 10.8 \\
B. thetaiotaomicron & 15.1 & 8 & 15.2 \\
B. ovatus & 3.6 & 3.6 & 6.1 \\
Others & $\cdots$ & $\cdots$ & \\
\hline
\end{tabular}


blood cultures and $69 \%$ in wounds in a survey in two military hospitals [10] to $54 \%$ in bacteroides infections of children [11]. B. thetaiotaomicron is usually the second most common species at $10-20 \%$ followed by $B$. vulgatus, $B$. distasonis and B. ovatus at $<10 \%$. The relative virulence of different Bacteroides spp. is not clearly understood, but has been ascribed to possession of a capsule [12], and resistance to serum complement [13-15] and to phagocytosis and intracellular killing $[16,17]$. The complexity of unravelling these pathogenic mechanisms has been reviewed recently for $B$. fragilis [18], and the importance of the chemistry of the capsular polysaccharide in the mechanism of abscess formation by $B$. fragilis has been demonstrated $[19,20]$. The lipopolysaccharides (LPS) of Bacteroides spp. vary in their biological activity (endotoxicity), with the LPS of $B$. fragilis being the most active in a range of models [21-23]. Most authors agree that $B$. fragilis is the most virulent species of the genus, and this was supported in the investigations cited above. Moreover, as the results of the present study did not confirm the previous suggestion that $B$. fragilis is particularly mucosaassociated, this indicates that the biological properties of this particular species of Bacteroides, rather than the fact that it is mucosa-adherent, are relevant to its pathogenic potential.

The mechanisms of virulence of the lesser pathogens of the genus are largely unexplored. The indication from this study that $B$. thetaiotaomicron is a possible inflammatory agent in UC does not correlate with the production of an LPS of high endotoxicity; the single strain of $B$. thetaiotaomicron investigated produced one of the least endotoxic of the LPS preparations in the model systems used [22], which suggests that other mechanism(s) may be operating.

Observations from parallel studies on the systemic [24] and local [25] antibody responses to Bacteroides LPS in health and disease are relevant to this study. Analysis of the local IgA response to $B$. fragilis and $B$. vulgatus in the colon, by measuring antibodies in a whole gut lavage fluid, has demonstrated that there is a major response to a common epitope within the LPS molecule of both species. Speculatively this may be a protective mechanism, neutralising potentially harmful endotoxin (LPS) within the healthy gut - the production of a cross-reactive antibody being much more efficient than multiple antibodies to all of the different serotype determinants [25]. Within the colon, the gut-associated lymphoid tissue may be multiply exposed to LPS from both the adherent and luminal (faecal) bacterial populations. Therefore, a response to common determinants might be more likely. However, a different mechanism appears to operate in the systemic immune system, where there is more evidence of the production of species-specific rather than cross-reactive anti-LPS IgG antibodies [24]. It is possible that individuals respond systemically more to the mucosa-associated populations than to the faecal organisms, because of proximity and ease of translocation.

These studies have shown the variation of the bacterial populations of the colonic mucosa and the antibody responses to them both locally and systemically within an individual patient. Some common themes are becoming apparent, but much more work is required to attempt to relate the observations to the mechanisms that operate to maintain homeostasis, and to further knowledge of the complex relationship between the normal bacterial flora and the local and systemic immune responses in the gastrointestinal tract in health and disease.

We thank donors to the Edinburgh Intestinal Immunology Research Fund and SmithKline Beecham for funding this research, Hannah Lough for skilled technical assistance, and the Scottish Anaerobe Reference Laboratory for providing facilities.

\section{References}

1. Duerden BI, Drasar BS (eds). Anaerobes in human disease. London, Edward Arnold. 1991.

2. Namavar F, Theunissen EBM, Verweij-Van Vught AMJJ et al. Epidemiology of the Bacteroides fragilis group in the colonic flora in 10 patients with colonic cancer. $J$ Med Microbiol 1989; 29: $171-176$.

3. Peach SL, Tabaqchali S. Mucosa-associated flora of the human gastrointestinal tract in health and disease. Eur $J$ Chemother Antibiot 1982; 2: 41-50.

4. Hudson MJ, Hill MJ, Elliott, PR, Berghouse LM, Burnham WR, Lennard-Jones JE. The microbial flora of the rectal mucosa and faeces of patients with Crohn's disease before and during antimicrobial chemotherapy. $J$ Med Microbiol 1984; 18 : $335-345$.

5. Hudson MJ. Anaerobes and inflammatory bowel disease. In: Borriello SP, Hardie JM (eds) Recent advances in anaerobic bacteriology. Dordrecht, Nijhoff. 1987: 60-71.

6. Hartley MG, Hudson MJ, Swarbrick ET, Grace RH, Gent AE, Hellier MD. Sulphasalazine treatment and the colorectal mucosa-associated flora in ulcerative colitis. Aliment Pharmacol Ther 1996; 10: 157-163.

7. McCourtie J, Poxton IR, Spence JA, Aitchison GU. Preliminary study of the anaerobic bacteria isolated from subgingival plaque from sheep. Vet Microbiol 1989; 21: 139-146.

8. Brown R, Collee JG, Poxton IR. Bacteroides, Fusobacterium and other Gram-negative rods; anaerobic cocci; identification of anaerobes. In: Collee JG, Fraser AG, Marmion BP, Simmons A (eds) Mackie and McCartney's Practical medical microbiology, 14th edn. New York, Churchill Livingstone. 1996: 501519.

9. Giaffer MH, Holdsworth CD, Duerden BI. The assessment of faecal flora in patients with inflammatory bowel disease by a simplified bacteriological technique. J Med Microbiol 1991; 35: $238-243$

10. Brook I. Recovery of anaerobic bacteria from clinical specimens in 12 years at two military hospitals. J Clin Microbiol 1988; 26: 1181-1188.

11. Brook I. Bacteroides infections in children. $J$ Med Microbiol 1995; 43: $92-98$

12. Onderdonk AB, Kasper DL, Cisneros RL, Bartlett JG. The capsular polysaccharide of Bacteroides fragilis as a virulence factor: comparison of the pathogenic potential of encapsulated and unencapsulated strains. J Infect Dis 1977; 136: 82-89.

13. Casciato DA, Rosenblatt JE, Bluestone R, Goldberg LS, Finegold SM. Susceptibility of isolates of Bacteroides to the bactericidal activity of normal human serum. I Infect Dis 1979; 140: 109-113.

14. Rotimi VO, Eke PI. The bactericidal action of human serum on Bacteroides species. J Med Microbiol 1984; 18: 355-363. 
15. Allan E, Poxton IR. The influence of growth medium on serum sensitivity of Bacteroides species. J Med Microbiol 1994; 41: 45-50.

16. Botta GA, Arzese A, Minisini R, Trani G. Role of structural and extracellular virulence factors in gram-negative anaerobic bacteria. Clin Infect Dis 1994; 18 Suppl 4: S260-S264.

17. Brook I. The role of encapsulated anaerobic bacteria in synergistic infections. FEMS Microbiol Rev 1994; 13 65-74.

18. Patrick S. The virulence of Bacteroides fragilis. Rev Med Microbiol 1993; 4: 40-49.

19. Tzianabos AO, Onderdonk AB, Rosner B, Cisneros RL, Kasper DL. Structural features of polysaccharides that induce intraabdominal abscesses. Science 1993; 262: 416-419.

20. Tzianabos AO, Onderdonk AB, Smith RS, Kasper DL Structure-function relationships for polysaccharide-induced intra-abdominal abscesses. Infect Immun 1994; 62: 3590-3593.
21. Poxton IR, Edmond DM. Biological activity of Bacteroides lipopolysaccharide - reappraisal. Clin Infect Dis 1995; 20 Suppl 2: S149-S153.

22. Delahooke DM, Barclay GR, Poxton IR. A re-appraisal of the biological activity of bacteroides lipopolysaccharide. $\mathrm{J} \mathrm{Med}$ Microbiol 1995; 42: 102-112.

23. Delahooke DM, Barclay GR, Poxton IR. Tumor necrosis factor induction by an aqueous phenol-extracted lipopolysaccharide complex from Bacteroides species. Infect Immun 1995; 63: 840-846.

24. Allan E, Poxton IR, Barclay GR. Anti-bacteroides lipopolysaccharide IgG levels in healthy adults and sepsis patients. FEMS Immunol Med Microbiol 1995; 11: 5-12.

25. Poxton IR, Myers CJ, Johnstone A, Drudy TA, Ferguson A. An ELISA to measure mucosal IgA specific for Bacteroides surface antigens in whole gut lavage fluid. Microbial Ecol Hlth Dis 1995; 8: 129-136. 